\title{
Comparison of a low transition temperature mixture (LTTM) formed by lactic acid and choline chloride with choline lactate ionic liquid and the choline chloride salt: physical properties and vapour-liquid equilibria of mixtures containing water and ethanol $\uparrow$
}

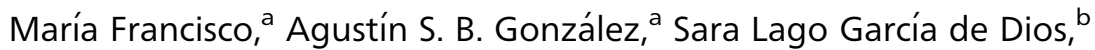 \\ Wilko Weggemans ${ }^{a}$ and Maaike C. Kroon ${ }^{\star a}$
}

A new group of nature-based solvents, i.e. low transition temperature mixtures (LTTMs), are evaluated in this work as entrainers for the separation of azeotropic mixtures. The effect of choline-based LTTMs on the vapour pressure of ethanol and water is compared to the effect of the corresponding lactate-based choline salt and a chloride-based choline salt. The changes with temperature and composition of the physical properties (density and viscosity) of the binary mixtures (entrainer + water-ethanol) are studied and compared. Also, the vapour-liquid equilibrium (VLE) data for binary mixtures of water-ethanol with the studied entrainers are reported. The phase diagrams of the ternary systems composed of water + ethanol + entrainer are shown and the experimental data are correlated by using the e-NRTL model.

Received 21st January 2013 Accepted 1st October 2013

DOI: $10.1039 / \mathrm{c} 3 \mathrm{ra} 40303 \mathrm{c}$

www.rsc.org/advances
Traditionally, very cheap and common salts were used as entrainers for azeotropic separations (water + alcohol) due to the well-known ebullioscopic effect, which strongly reduces the volatility of the water. ${ }^{6}$ However, salts show multiple disadvantages for large scale processes: they may erode the sieve plate, precipitate and build up in the tower due to causticity and limited solubility.

Extractive distillation with ionic liquids (ILs) as separating agents became very popular during the past decade. ${ }^{7-9}$ The use of these ionic substances integrates the advantages of a liquid solvent (easy operation) and solid salt (high separation ability).

Another advantage of these entrainers is their negligible vapour pressure near ambient conditions. This minimizes the chance of solvent emissions to the atmosphere, thus reducing the atmospheric pollutant effect of volatile solvents.

However, the scientific community started questioning the use of ILs for large scale applications. The synthesis of most ILs is not "green" and the costs of production as well as degree of recyclability or biodegradability are the main weak points of IL technologies. ${ }^{\mathbf{1 0 , 1 1}}$

A new family of solvents was presented for the first time by Abbott et al. as suitable alternative to ILs. ${ }^{12}$ Originally, they were called "deep eutectic solvents" (DES), ${ }^{12}$ but this name does not cover the complete class of solvents, because many of them do not show (eutectic) melting points but glass transitions instead. ${ }^{13}$ Therefore, we coined them "low transition temperature mixtures" (LTTM). ${ }^{13}$ These new solvents can be formed by mixing two solid starting materials, which form a liquid by 
hydrogen bond interactions. They show very interesting properties as solvents to be explored for different applications in separation processes. They share many advantages with ILs, but their preparation is simple and cheap, since one only has to mix natural and readily available starting materials. ${ }^{\mathbf{1 4 , 1 5}}$ LTTMs are formed by mixing a hydrogen bond donor (e.g. organic acid, alcohol) and a hydrogen bond acceptor (e.g. quaternary ammonium salt). One of the most popular hydrogen bond acceptors used to form LTTMs is choline chloride, because of its benign character, biodegradability and price.

In our previous work, ${ }^{16}$ lactic acid:choline chloride mixtures were investigated as solvents for separations. A complete characterization of the physical properties proved the tunability and phase behaviour by changing the composition. These mixtures formed a stable liquid in a wide range of compositions. Their promising properties as solvents, like wide liquid range, good salvation properties derived from hydrogen bonding interactions, recoverability and biodegradability, make lactic acid:choline chloride mixtures promising candidates as solvents for separations. ${ }^{16}$

In this work, we choose a representative LTTM formed by lactic acid and choline chloride in a molar ratio $2: 1$ (LC2 : 1), and we compare its performance as entrainer in extractive distillation with the choline chloride salt and a lactate-based IL (choline lactate). For this purpose, we study the properties of binary mixtures of these three entrainers with water and ethanol in order to discuss the interactions involved. The VLE data of binary mixtures of LTTM (LC2 : 1), IL (choline lactate) and salt (choline chloride) with water and ethanol are also studied, as well as the azeotropic behaviour of the ternary system of ethanol + water + entrainer. The structures and phase transition temperatures of the three different entrainers studied are shown in Fig. 1.

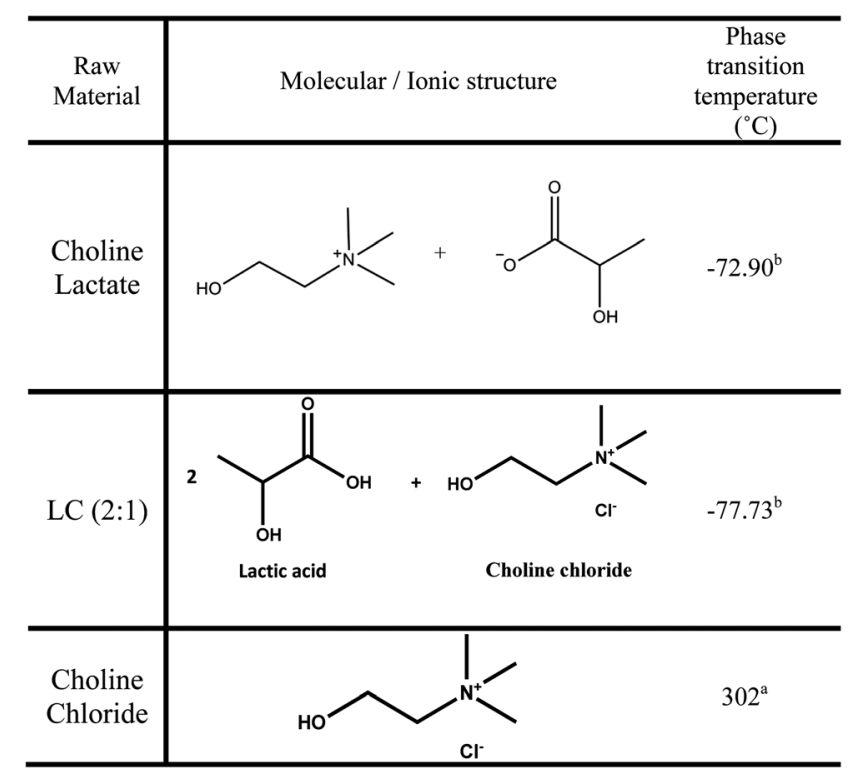

Fig. 1 Molecular structure of the three entrainers studied in this work (the IL choline lactate, the LTTM formed by lactic acid and choline chloride (molar ratio $2: 1$ ), and the natural salt choline chloride) and their respective phase transition temperatures. ( ${ }^{a}$ Melting point. ${ }^{b}$ Glass transition temperature.)

\section{Results and discussion}

\section{Physical properties: density, viscosity and mixing properties}

In the field of separation processes, the study of the physical properties of the mixtures involved in a process is of crucial interest. In this way, the suitability of a solvent for a certain process can be evaluated and the design of the involved units can be made. Therefore, the characterization of the main physical properties of the binary mixtures composed of the solvent to be used as entrainer and water or ethanol are determined here. The deviation of the ideal behaviour of these mixtures is also discussed.

\section{Properties of binary mixtures with water}

Density and excess molar volume. The densities of binary mixtures of water with the IL choline lactate, the LTTM LC2 : 1, or the salt choline chloride, respectively, are studied as a function of temperature and composition. Fig. 2 shows the dependency of the density on temperature for all three cholinederived components studied in this work at two representative mole fractions of entrainer (0.1 and 0.3). The experimental values for the three binary systems are included in the ESI (Table S1†).

The density follows a linear trend with temperature as expressed in eqn (1):

$$
\ln (\rho)=A+B(T)
$$

where $\rho$ is the density in $\mathrm{g} \mathrm{cm}^{-3}$ and $T$ is the temperature in $\mathrm{K}$.

The fitting parameters of eqn (1) obtained at different molar compositions of the entrainer + water binary mixtures, including the deviations between the calculated and the experimental density values, are reported as ESI (Table S2 $\dagger$ ).

The study of molar excess volume of the binary mixtures and their change with temperature and composition is very useful for a better understanding of the interactions involved in the mixing behaviour of real solutions. The experimental values of excess molar volume, $V_{\mathrm{m}}^{\mathrm{E}}\left(\mathrm{cm}^{3} \mathrm{~mol}^{-1}\right)$ of the mixtures were

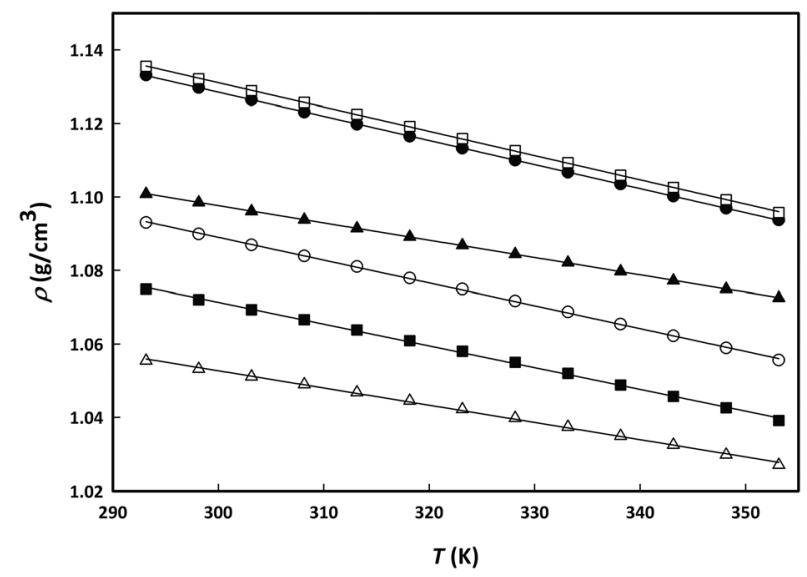

Fig. 2 Linear density change with temperature for aqueous binary mixtures of choline lactate $\left(\mathrm{O}, x_{\mathrm{IL}}=0.1002 ; \bullet, x_{\mathrm{LL}}=0.2990\right), \mathrm{LC2}: 1\left(\mathbf{\square}, x_{\mathrm{LC2}}:{ }_{1}=0.1003\right.$; $\left.\square, x_{\mathrm{LC} 2}:_{1}=0.2944\right)$ and choline chloride $\left(\triangle, x_{\text {SALT }}=0.0991 ; \mathbf{\Delta}, x_{\text {SALT }}=0.3033\right)$. 
calculated from the measured densities of binary mixtures using the following expression:

$$
V_{\mathrm{m}}^{\mathrm{E}}=\sum_{i} x_{i} M_{i}\left(\rho^{-1}-\rho_{i}^{-1}\right)
$$

where $x_{i}$ and $M_{i}$ are the mole fraction and molar weight of the pure components in the mixture, and $\rho$ and $\rho_{i}$ are the experimental density of the mixture and the pure components, respectively. At these temperatures, the salt choline chloride is solid so its density in solid state was used to calculate the $V_{\mathrm{m}}^{\mathrm{E}}$ of its binary mixtures.

Fig. 3 presents the experimental excess molar volumes, $V_{\mathrm{m}}^{\mathrm{E}}$, of the three binary water + entrainer (IL/LTTM/salt) systems as a function of molar fraction at two representative temperatures ( 293.15 and $348 \mathrm{~K}$ ). All experimental data can be found in the ESI (Table S3†).

The experimental $V_{\mathrm{m}}^{\mathrm{E}}$ data were fitted by the often used Redlich-Kister equation ${ }^{17}$ described as follows:

$$
V_{\mathrm{m}}^{\mathrm{E}}=x_{1}\left(1-x_{1}\right) \sum_{i} A_{i}(T)\left(2 x_{1}-1\right)^{i-1}
$$

where:

$$
A_{i}(T)=b_{i}+c_{i}(T)
$$

where $x_{1}$ is the mole fraction of the entrainer and $V_{\mathrm{m}}^{\mathrm{E}}$ $\left(\mathrm{cm}^{3} \mathrm{~mol}^{-1}\right)$ is the molar excess volume. The values of the parameters $\left(A_{i}\right)$ have been determined using the method of least-squares. The fitting parameters are summarized in Table $\mathrm{S} 4 \dagger$ together with the deviations found for this correlation.

From Fig. 3 it can be observed that larger negative deviations from ideality are found at lower temperatures and higher water concentrations. This graph also depicts the strong asymmetrical behaviour of the excess molar volumes with composition. The minimum value of $V_{\mathrm{m}}^{\mathrm{E}}$ is found at mole fraction close to 0.3 and is slightly shifting to lower values with increasing temperatures. The negative $V_{\mathrm{m}}^{\mathrm{E}}$ values are indicative of a highly

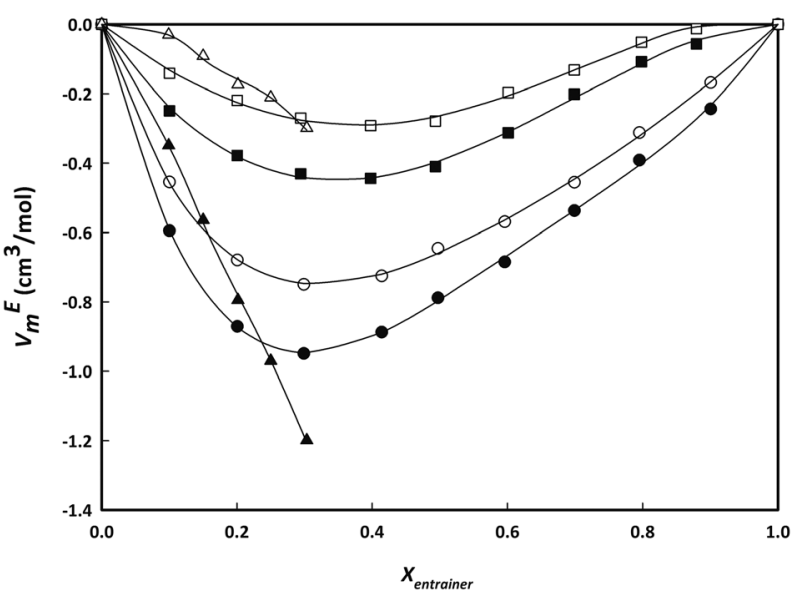

Fig. 3 Experimental excess molar volume $V_{\mathrm{m}}^{\mathrm{E}}\left(\mathrm{cm}^{3} \mathrm{~mol}^{-1}\right)$ of aqueous binary mixtures of choline lactate (circles), LC2: 1 (squares) and choline chloride (triangles) as a function of composition for two representative temperatures (293.15 K, black; $348.15 \mathrm{~K}$, white). Lines represent the fitting with Redlich-Kister equation (3). attractive interaction with water. The IL or salt + water mixtures show a larger deviation from ideality compared to the $\operatorname{LC}(2: 1)+$ water mixture.

Viscosity and viscosity deviation. The experimental values of the dynamic viscosity for the binary mixtures of $\{\mathrm{IL} / \mathrm{LTTM} / \mathrm{salt}$ (1) + water (2)\} are reported as a function of temperature and composition and included as ESI (Table S5†). Fig. 4 shows the viscosity change with temperature for the three studied aqueous binary mixtures at the same mole fraction of entrainer $(0.3)$.

The viscosity dependency on temperature was successfully fitted to the Vogel-Fulcher-Tamman (VFT) equation (5), which was previously applied for ILs: ${ }^{18}$

$$
\eta=A \exp \left(\frac{B}{T-T_{0}}\right)
$$

where $A, B$ and $T_{0}$ are the fitting parameters obtained by linearization of the equation. The parameters and deviations for the fitting of the experimental data are included as ESI in Table S6 ${ }^{\dagger}$ and the fit is shown as solid lines in Fig. 4.

From Fig. 4, it can be observed that viscosity of the IL + water mixture is highest, followed by the salt + water mixture, and the viscosity of the $\operatorname{LC}(2: 1)+$ water mixture is the lowest. The same trend (IL > salt > LTTM) was found for the dependency of the viscosity on the temperature; the IL viscosity being most influenced by temperature.

The deviation in the viscosity for the binary mixtures, $\Delta \eta$, was obtained from the relation:

$$
\Delta \eta=\eta-\sum_{i}\left(x_{i} \eta_{i}\right)
$$

where $\eta$ is the absolute dynamic viscosity of the mixture, and $\eta_{i}$ are the viscosities of the pure components.

The experimental values of $\Delta \eta$ for the binary aqueous mixtures of the IL choline lactate and the LTTM LC2 $: 1$ are displayed as ESI in Table S7. $\uparrow$ The viscosity deviation of the salt + water mixture cannot be determined because the pure salt is a solid with infinite viscosity. The experimental values were fitted

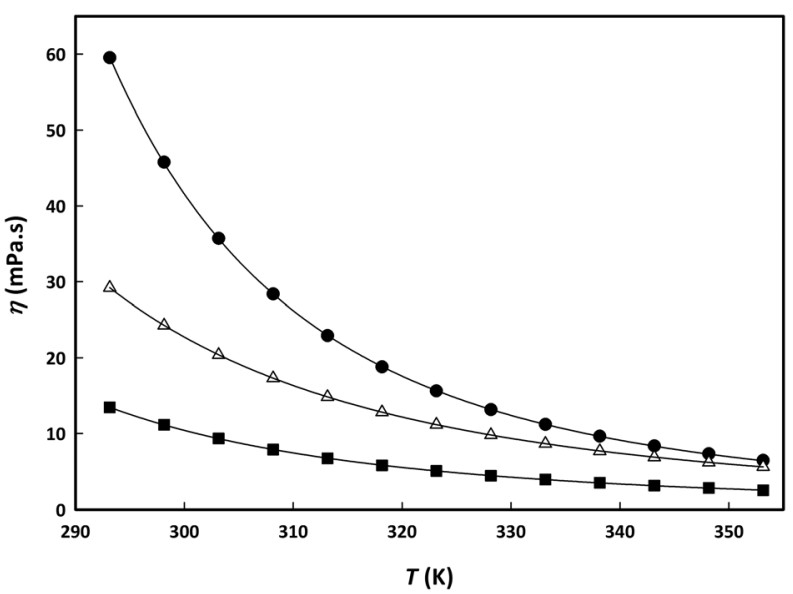

Fig. 4 Viscosity change with temperature for aqueous binary mixtures of choline lactate (circles), LC2: 1 (squares) and choline chloride (triangles) at the same molar fraction (0.3). Lines represent fitting of the experimental data using VFT equation (5). 
to a Redlich-Kister polynomial equation similar to the one used for excess molar volumes:

$$
\Delta \eta=x_{1}\left(1-x_{1}\right) \sum_{i} A_{i}(T)\left(2 x_{1}-1\right)^{i-1}
$$

where $A_{i}$ is defined by eqn (4).

The fitting parameters are summarized in Table S8 together with the deviations defined in Table S2. $\dagger$ Fig. 5 shows the experimental data and fitting curves for two representative temperatures (293.15 and $318.15 \mathrm{~K})$. In all cases, the deviation in viscosity is negative. For the IL + water system, the minimum in viscosity deviation can be found at a mole fraction of approximately 0.5 , while the minimum for the LTTM + water system is present at a mole fraction of 0.7 . For both systems, the viscosity deviation decreases as the temperature increases; this behaviour is similar compared with other systems found in literature..$^{18}$

Fig. 6 shows an interesting comparison between the change in properties of aqueous binary mixtures of IL and LTTM at a mole fraction of 0.5 as a function of temperature. More negative values were found for the excess molar volume of the IL choline lactate compared to those of the LTTM LC2:1, while the viscosity deviations were found to be lower in the whole range of temperatures. The addition of water thus has a stronger effect on the LTTM viscosity while the presence of water has a larger effect on the density of the IL. ${ }^{19}$

\section{Properties of binary mixtures with ethanol}

In this section, a comparative study is made between the properties of the binary mixture of the IL choline lactate with ethanol and the $\mathrm{IL}+$ water system (in the previous section).

Similar conclusions would be obtained for comparison of the other choline-based compounds with water and ethanol, so only one of the binary mixtures with ethanol is discussed.

Density and excess molar volume. The experimental densities of the binary mixtures of the IL choline lactate and ethanol

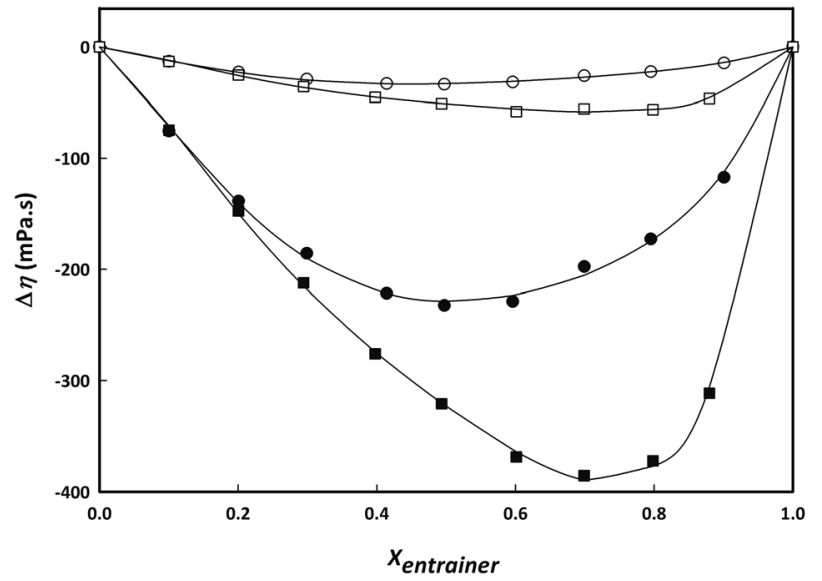

Fig. 5 Viscosity deviations of aqueous binary mixtures of choline lactate (circles) and LC2:1 (squares) as a function of composition for two representative temperatures (293.15 K, black; 318.15 K, white). Lines represent fitting with Redlich-Kister equation (7).

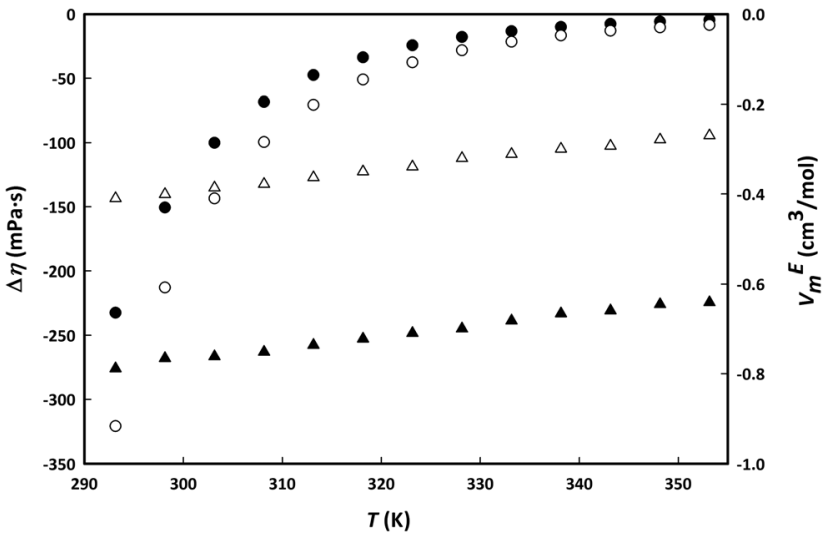

Fig. 6 Viscosity deviations, $\Delta \eta$ (circles) and excess molar volume, $V_{\mathrm{m}}^{\mathrm{E}}$ (triangles) for aqueous mixtures of 0.5 molar fraction of binary mixtures of choline lactate (black) and LC2 : 1 (white) with water.

are included as ESI (Table S9†). The density change with temperature follows the expected linear trend described by eqn (1). The fitting parameters as well as the corresponding deviations of the experimental values are also included as ESI (Table S10 $\dagger$ ). Table S11 $\dagger$ shows the experimental excess molar volumes for the binary mixtures of IL and ethanol calculated from the experimental densities following eqn (2). The RedlichKister equation (3) is used for the fitting of the experimental data. The fitting parameters and deviations are included in Table S12.†

Viscosity and viscosity deviation. The experimental data for viscosities and the fitting parameters of VFT equation (5) fitting, as well as their deviations are included in ESI (Tables S13 and $14 \dagger)$. Table $\mathrm{S} 15^{\dagger}$ shows the deviations in viscosity calculated from the experimental data by using eqn (6). The Redlich-Kister equation (7) is again used for the correlation of the experimental data. The fitting parameters and deviations are included in Table S16. $\dagger$

Fig. 7 shows a comparison between the deviations from ideal behaviour (represented by $V_{\mathrm{m}}^{\mathrm{E}}$ and $\Delta \eta$ ) for the IL mixtures with ethanol and water at a temperature of $293.15 \mathrm{~K}$. Both show similar behaviour with negative deviations for both properties pointing to stronger interactions of IL-water-ethanol compared to cation-anion interactions. In both cases the minimum of the curve is located at the same IL composition, which can be interpreted as a similar solvation number for the aggregation state of cation/anions in water or ethanol.

\section{Vapour-liquid equilibria}

Vapour pressures of binary mixtures with water and ethanol. In this section, a comparative study is made between the vapour-liquid equilibrium data of binary mixtures of the IL choline lactate, the LTTM LC2 : 1 and the salt choline chloride with water and ethanol.

The change in vapour pressure of water in the presence of each one of the choline-based entrainers is studied first. These data are useful for a comparison of the effect of the addition of entrainer on the activity coefficient of water. Table 1 reports the 


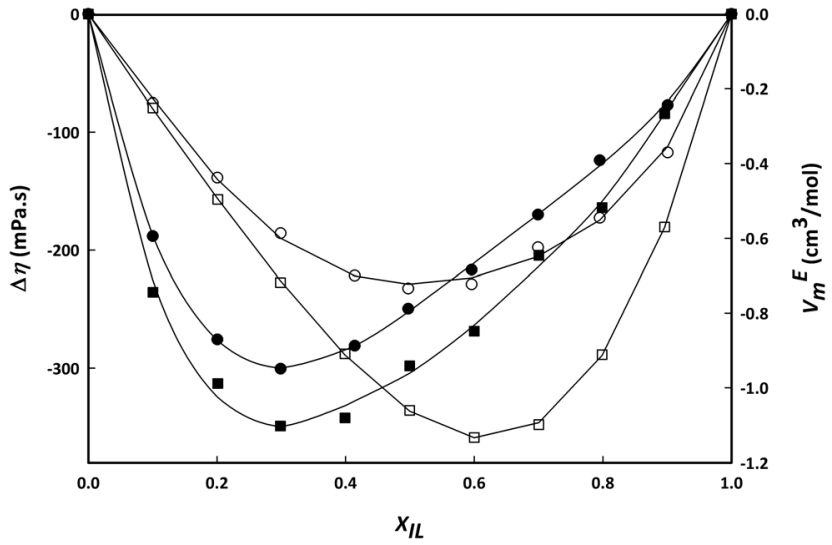

Fig. 7 Viscosity deviations, $\Delta \eta$ (white) and excess molar volume, $V_{\mathrm{m}}^{\mathrm{E}}$ (black) for binary mixtures of choline lactate and water (circles) or ethanol (squares) at 293.15 K.

VLE experimental data for the binary mixtures of water with the three entrainers. The Antoine equation ${ }^{20}$ was used to correlate the vapour pressure data:

$$
\ln \left(P_{i}^{0} / \mathrm{Pa}\right)=A_{i}-\frac{B_{i}}{T / \mathrm{K}+C_{i}}
$$

where $P^{0}$ is the pure component vapour pressure, $T$ is the temperature, and $A_{i}, B_{i}$, and $C_{i}$ are the Antoine parameters.

The Antoine parameters of eqn (8) and the deviations between the correlation and the experimental data for the studied mixtures are included as ESI (Tables S17 and S18 $\dagger$ ).

Fig. 8 shows a comparison in the change of the vapour pressure of water by addition of the IL choline lactate, the LTTM LC2 : 1 and the salt choline chloride. The presence of the salt shows the highest effect on the vapour pressure of water compared to the IL and the LTTM. Reason for the higher effect of the salt compared to the IL is the difference in the strength of the hydrogen bond interaction between the anion and the water. Higher interactions are expected for the chloride ion due to its higher charge/size ratio and, therefore, its higher ability for polarization. The lowest effect on the vapour pressure was found for the LTTM LC2 : 1 using the same molar ratio. Because only $1 / 3$ of the moles of LC2 : 1 consists of choline chloride (and $2 / 3$ of lactic acid), the LTTM only has a maximum of $1 / 3$ of the salt's ebullioscopic effect. This point could evidence that the ability of the LTTM to affect the vapour pressure of water is mainly determined by the ebullioscopic effect of the constituent salt. Further studies are required to confirm this conjecture.

Table 2 shows the VLE data for binary mixtures with ethanol. A similar plot to the one discussed above could be made for the

Table 1 VLE data for binary mixtures composed of (i) choline chloride + water,(ii) $L C(2: 1)+$ water and (iii) choline lactate + water

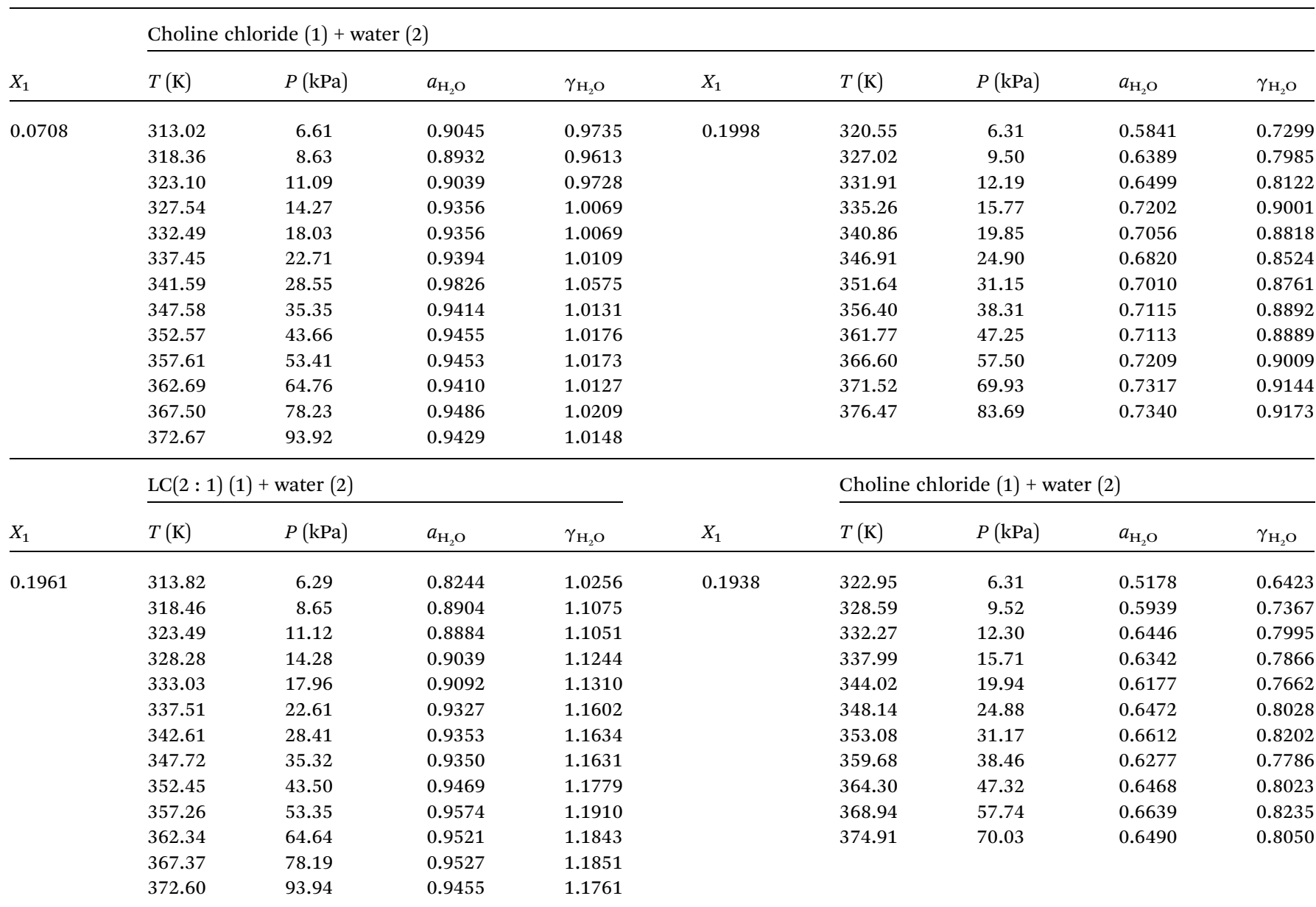




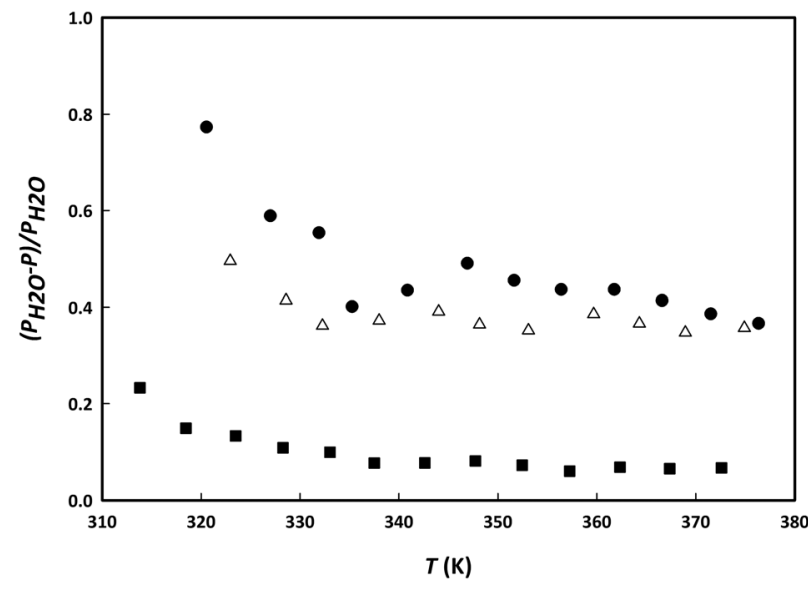

Fig. 8 Relative water vapour pressure change by addition of the IL choline lactate (triangles), the LTTM LC2 : 1 (squares) and the salt choline chloride (circles)

binary mixtures with ethanol, but the relative change in vapour pressure is close to zero in all cases. This difference in behaviour points to a much higher effect of the entrainers on the volatility of water compared to that of ethanol. It would be, in principle, reasonable to assume that LTTMs (just like ILs and salts) could be useful entrainers for water-ethanol separation by increasing their relative volatility and so, breaking the azeotropic behaviour. However, the effect of the IL on the relative volatility is much higher $(3 \times)$ compared to the LTTM. Therefore, in the next section, only ternary VLE data are measured for the IL + water + ethanol system.

VLE of ternary system of choline lactate + ethanol + water. Table 3 shows the VLE data of ternary system consisting of the ethanol (1) + water (2) + choline lactate (3) at $100 \mathrm{kPa}$, obtained by keeping the IL mole fraction nearly constant at $x_{3} \approx 0.2$. In this table, $x_{3}$ is the mole fraction of IL in the liquid phase, $x_{1}{ }^{\prime}$ is the mole fraction of ethanol in the liquid phase expressed on an IL-free basis, $y_{1}$ is the mole fraction of ethanol in the vapour phase, and $T$ is the equilibrium temperature. The relative volatility, $\alpha^{\mathrm{VLE}}$, and selectivity, $S^{\mathrm{VLE}}$, can be calculated from the VLE data as follows:

$$
\alpha_{i j}^{\mathrm{VLE}}=\frac{\left(y_{i} / x_{i}\right)}{\left(y_{j} / x_{j}\right)}
$$

Table 3 VLE data for ternary mixtures of ethanol (1) + water (2) + choline lactate (3) ternary system, at $100 \mathrm{kPa}$

\begin{tabular}{llllll}
\hline$x_{3}$ & $x_{1}{ }^{\prime}$ & $y_{1}$ & $T(\mathrm{~K})$ & $\alpha_{12}^{\mathrm{VLE}}$ & $S_{12}^{\mathrm{VLE}}$ \\
\hline 0.2031 & 0.000 & 0.000 & 378.79 & - & - \\
0.2000 & 0.031 & 0.080 & 369.23 & 2.707 & 0.447 \\
0.2000 & 0.063 & 0.197 & 366.12 & 3.677 & 0.641 \\
0.2000 & 0.125 & 0.367 & 361.27 & 4.070 & 0.798 \\
0.2000 & 0.156 & 0.458 & 359.66 & 4.557 & 0.991 \\
0.2000 & 0.195 & 0.506 & 357.01 & 4.228 & 1.235 \\
0.2000 & 0.328 & 0.630 & 352.10 & 3.496 & 1.275 \\
0.2009 & 0.438 & 0.697 & 351.61 & 2.957 & 1.640 \\
0.2008 & 0.500 & 0.740 & 351.53 & 2.844 & 1.900 \\
0.2007 & 0.563 & 0.774 & 351.24 & 2.668 & 1.936 \\
0.2007 & 0.687 & 0.833 & 351.18 & 2.273 & 2.324 \\
0.2007 & 0.842 & 0.925 & 351.17 & 2.307 & 2.270 \\
0.2007 & 0.875 & 0.940 & 351.11 & 2.254 & 2.065 \\
0.2007 & 0.906 & 0.952 & 351.06 & 2.050 & 1.607 \\
0.2009 & 0.938 & 0.960 & 351.05 & 1.595 & 1.313 \\
0.2008 & 0.969 & 0.976 & 351.03 & 1.304 & 0.447 \\
0.2009 & 1.000 & 1.000 & 351.01 & - & - \\
\hline
\end{tabular}

$$
S^{\mathrm{VLE}}=\frac{\alpha_{i j}^{\text {with entrainer }}}{\alpha_{i j}^{\text {without entrainer }}}
$$

Fig. 9 shows the evolution of the azeotrope with the addition of choline lactate as entrainer at different molar fractions of IL, while Fig. 10 shows the activity coefficients of ethanol and water as a function of ethanol composition. The electrolyte nonrandom two-liquid (e-NRTL) model is used to correlate the VLE data. This model is an extension of the nonrandom two-liquid local composition proposed by Renon and Prausnitz ${ }^{21}$ for liquid-phase activity coefficients. These activity coefficients can be obtained by adjusting the VLE data of the ternary system through the minimization of the objective function $\mathrm{OF}$ described as follows:

$$
\mathrm{OF}=\sum_{N}\left(1-\frac{\gamma_{\text {1cal }}}{\gamma_{\text {1exp }}}\right)^{2}+\left(1-\frac{\gamma_{2 \text { cal }}}{\gamma_{2 \exp }}\right)^{2}
$$

where $\gamma_{i}$ is the activity coefficient of solvent $i$. Following this procedure, it is possible to obtain the binary parameters

Table 2 VLE data for binary mixtures composed of (i) choline lactate + ethanol and (ii) $L C(2: 1)+$ ethanol

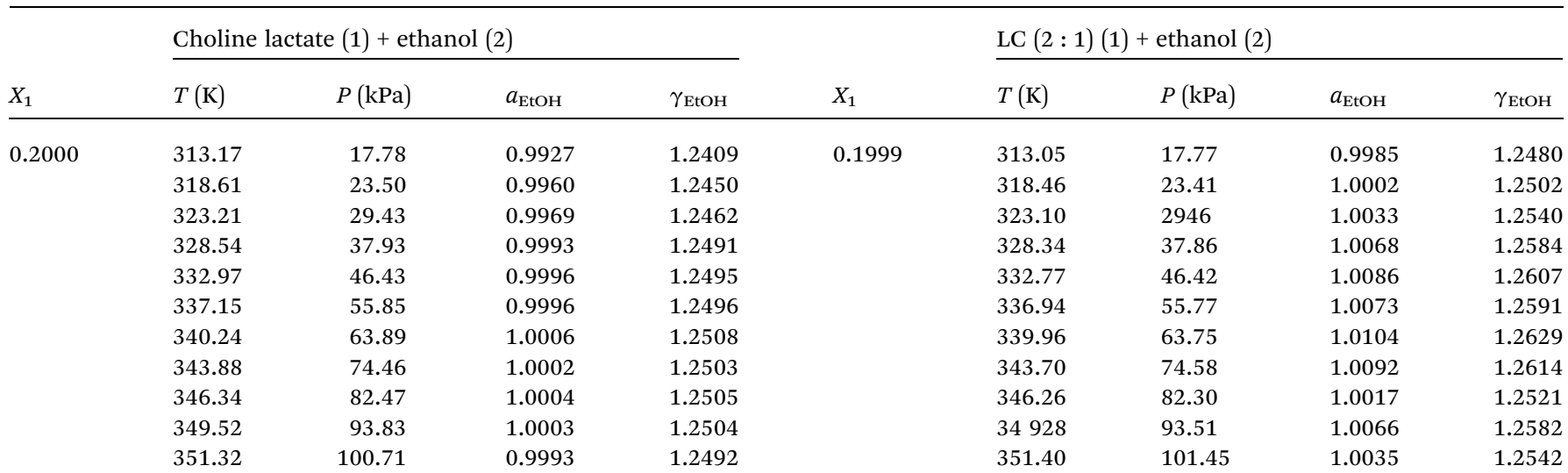




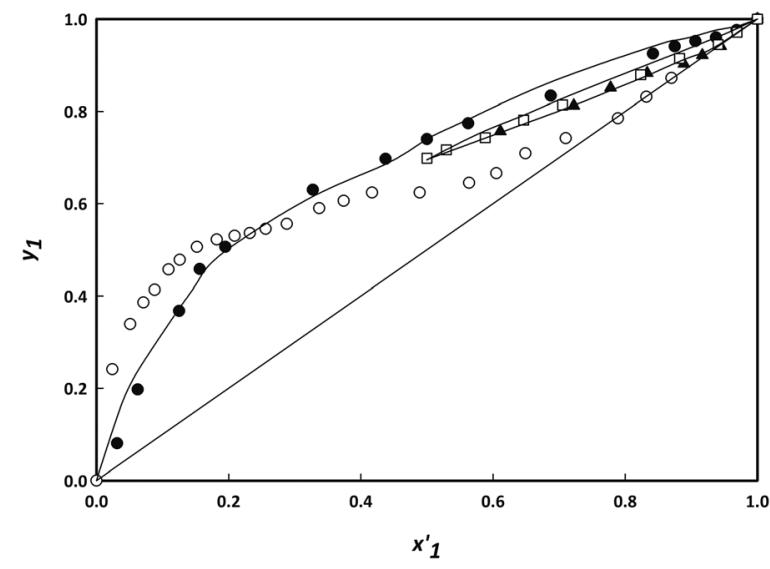

Fig. 9 Salting out effect of choline lactate on the VLE of the ethanol (1) + water (2) system at $100 \mathrm{kPa}$ for IL mole fraction of $0.2(\bullet), 0.15(\square)$ and $0.1(\mathbf{\Lambda})$ [this work] compared to IL-free system $(O) .{ }^{22}$ Solid lines represent fitting of the experimental data using e-NRTL model.

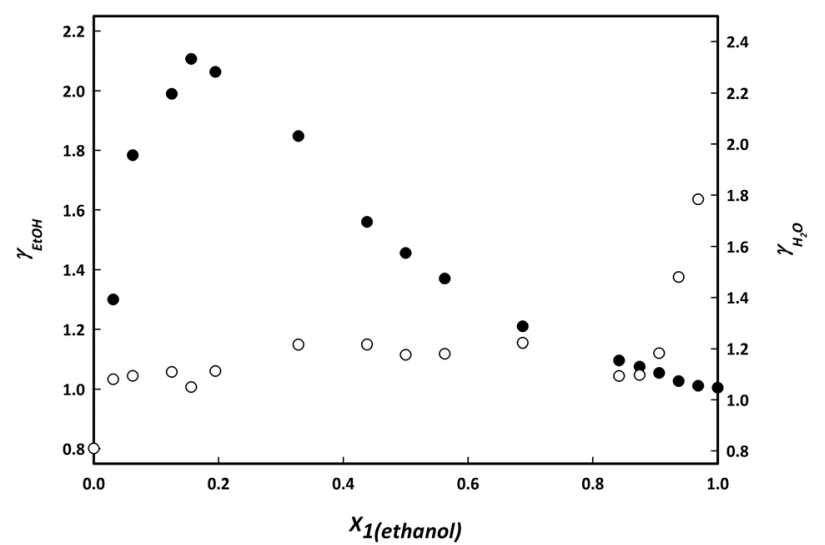

Fig. 10 Activity coefficients $\gamma$ of ethanol (black) and water (white) in the ternary mixture with 0.2 mole fraction of IL.

IL-water and IL-ethanol by assuming ideal behaviour of the vapour phase and iteratively solving the equilibrium conditions expressed in eqn (12) for the solvent:

$$
y_{i} p=X_{i} \gamma_{i} P_{i}^{0}
$$

where $y_{i}$ is the vapor-phase mole fraction of solvent $i ; p$ is the total pressure in the system; $X_{i}$ is the liquid-phase mole fraction based on the assumption of total dissociation of electrolytes; $\gamma_{i}$ is the activity coefficient of component $i$ obtained from the eNRTL model; and $P_{i}^{0}$ is the vapour pressure of solvent $i$ at equilibrium temperature, which can be calculated using the Antoine coefficients. The binary interaction parameters for the e-NRTL model and the deviations of the model with the experimental data are included as ESI (Table S19†).

Using the relative volatility as separation factor at an ethanol mole fraction of 0.95 and $100 \mathrm{kPa}$, most of the ionic liquids achieve an average relative volatility of $1.33 .{ }^{23-26}$ As can be seen in Table 3, the relative volatility of ethanol when using choline lactate is in agreement with this average value.
Fig. 10 shows how the presence of the IL changes the relative volatility of the ethanol + water system. The binary system in absence of IL shows azeotropic behaviour. Addition of the IL results in an azeotropic composition shift to higher concentrations of ethanol (as observed for the mixture containing 0.10 and 0.15 mole fractions of IL) until the azeotrope completely disappears at a mole fraction of 0.2 .

\section{Conclusions}

In this work, the use of a LTTM (mixture of lactic acid + choline chloride in the ratio $2: 1$ ) as possible entrainer for azeotropic distillation was evaluated and compared to a lactate-based IL (choline lactate) and the choline chloride salt. The main interactions between these entrainers with the azeotropic mixture ethanol + water are discussed. The strong negative values found for the deviations of their properties from ideal behaviour point to strong hydrogen-bond interactions between the entrainer and ethanol-water. The vapour pressure of water was strongly reduced by addition of all three entrainers. The highest effect was found for the salt choline chloride. The salting out effect of the LTTM was found to be lower caused by the lower concentration of choline chloride in the mixture. The study of the azeotropic behaviour of the ternary IL + ethanol + water shows a shift of the azeotropic point to higher concentrations of ethanol upon the addition of the IL, until the azeotrope completely disappears at a IL mole fraction of 0.2.

\section{Experimental section}

\section{Chemicals}

The IL choline lactate with a purity of $>98 \mathrm{wt} \%$ was supplied by Iolitec, synthesized on request. It was used without any further purification. Ethanol ( $\geq 99.5 \mathrm{wt} \%$ ) was purchased from TechniSolv. Ultra-pure crystalline DL-lactic acid at pharmaceutical grade was kindly provided by PURAC Biochem B.V. (Gorinchem, Netherlands). Choline chloride was obtained from Aldrich ( $\geq 98$ wt $\%$ ). Deionized MilliQ water was used in all the studied mixtures $(<18.2 \mathrm{M} \Omega \mathrm{cm})$. Choline lactate, choline chloride and lactic acid were dried under vacuum before use. The water content was measured by using Karl Fischer (KF796) titration to be $<1 \mathrm{wt} \%$ in all experiments.

\section{Preparation of LTTM}

LTTM formed by mixing lactic acid and choline chloride were prepared following a similar procedure previously reported by our group. ${ }^{13}$ The mixtures were weighted using a Mettler AX205 balance with a precision of $\pm 0.02 \mathrm{mg}$, whereby the water content of the starting materials was always measured and taken into account. Both the hydrogen bond donor (lactic acid) and acceptor (choline chloride) were added to a closed $25 \mathrm{~mL}$ glass flask and homogeneously mixed before heating. The temperature was set to $60 \pm 0.1{ }^{\circ} \mathrm{C}$ using a thermostatic oil bath with a IKA ETS-D5 temperature controller. Once a transparent liquid was formed, the mixture was cooled down. In our previous works, ${ }^{13,15}$ a TGA analysis (TGA Q500 by TA Instruments) was carried out to check the thermal stability at a scan 
rate of $10{ }^{\circ} \mathrm{C} \min ^{-1}$ under a nitrogen flow with a weighing precision of $\pm 0.01 \%$ a sensitivity of $0.1 \mu \mathrm{g}$, and an isothermal temperature precision of $\pm 0.1{ }^{\circ} \mathrm{C}$. Differential Scanning Calorimetry (DSC) Q1000 was used to obtain the glass transition temperature of the formed LTTM with a scan rate of $10{ }^{\circ} \mathrm{C}$ $\min ^{-1}$ a sensitivity of $0.2 \mu \mathrm{W}$ and a temperature precision of $\pm 0.1^{\circ} \mathrm{C}$. The water content was measured with the Karl-Fischer titration method on a Metrohm $870 \mathrm{KF}$ Titrino plus.

\section{Determination of physical properties: density and viscosity}

The density and the viscosity of the three entrainers were determined at atmospheric pressure and at temperatures ranging from 298 to $353 \mathrm{~K}$. Due to the high hygroscopicity of the samples, both properties were measured using an Anton Paar SVM 3000/G2 Stabinger viscometer. This equipment requires only a total volume of sample of $2.5 \mathrm{~mL}$, which is not in contact with the atmosphere during the measurement, so that moisture capture from the air is avoided. The equipment is provided with a high-precision thermostat with a stability of $0.005 \mathrm{~K}$ and calibrated by the manufacturer. The uncertainties of the density (with viscosity correction) and viscosity measurements are $\pm 0.0005 \mathrm{~g} \mathrm{~cm}^{3}$ and $\pm 0.35 \%$ respectively.

\section{Determination of VLE}

An all-glass ebulliometer (Fischer VLE 602D) was used for the determination of the experimental VLE data. In this device, the equilibrium chamber is a dynamic recirculating still, equipped with a Cottrell circulation pump. The operation procedure is based on the principle of the circulation method. ${ }^{27}$ A control unit (Fischer VLE 2+) was used to set the pressure and heating power in the immersion heater. The equilibrium was reached after 30 minutes or longer, when the condensation rate (12 drops per second) and equilibrium temperature were constant in order to ensure a stationary state. Pressure and equilibrium temperature could be determined with an accuracy of $\pm 0.01 \mathrm{kPa}$ and $\pm 0.01 \mathrm{~K}$, respectively. Two or three replicate runs were performed for each sample.

All solutions were prepared gravimetrically with an electronic balance (Mettler Toledo AX 205) with a readability of $\pm 10^{-4} \mathrm{~g}$. VLE data of 5 binary systems were measured i.e., water + IL, water + LTTM, water + salt, ethanol + IL and ethanol + LTTM. The salt choline chloride was found to be immiscible with ethanol. The VLE data of the ternary system consisting of water + ethanol + IL were also measured. Mixtures of water + IL or ethanol + IL were added to the initial sample in order to maintain a constant IL mole fraction in the system.

Samples of the condensed vapour phase were periodically analyzed by gas chromatograph (GC) and High Pressure Liquid Chromatography (HPLC, Varian Prostar) in order to guarantee the absence of entrainer in the vapour phase.

Vapour phase samples were injected in a Varian Prostar HPLC equipped with a silica-based Grace Prevail ${ }^{\mathrm{TM}}$ Organic Acid column $(250 \mathrm{~mm} \times 4.6 \mathrm{~mm})$ with a particle size of $5 \mu \mathrm{m}$. The temperature of the column was kept constant in an oven (Varian Prostar Model 510) at 313.2 K for all measurements. Detection of entrainers was done using a UV detector (Varian Prostar model 310) at $210 \mathrm{~nm}$. Each sample was injected twice.

The amount of ethanol in the condensed vapour phase was analysed using a Varian CP-3800 gas chromatograph (GC) equipped with a flame ionization detector $\left(250^{\circ} \mathrm{C}\right)$ and a Varian CP-SIL 5CB column $(25 \mathrm{~m} \times 1.2 \mu \mathrm{m})$. The injector temperature was $275{ }^{\circ} \mathrm{C}$ and the carrier gas was helium with a constant flow rate of $4.0 \mathrm{~mL} \mathrm{~min}^{-1}$. The water mole fraction was obtained from a mass balance calculation. Due to the negligible volatility of the salt/IL/LTTM, no presence of any of the studied entrainers was detected in the vapour phase. With this method, the detection limits for ethanol and lactic acid are, respectively, 0.003 and 0.0002 in mole fraction.

The liquid phase of the ternary system is composed of ethanol, water and the IL choline lactate. The ethanol mole fraction in the liquid phase was determined using the same GC, while the water content in the liquid phase was analysed using Karl-Fischer titration on a Metrohm $870 \mathrm{KF}$ Titrino plus. The concentration of all components in both phases could be determined with an average overall deviation in mole fraction of \pm 0.004 .

\section{Notes and references}

1 A. Pandey, Handbook of Plant-based Biofuels, CRC, 2008.

2 V. Gomis, R. Pedraza, O. Francés, A. Font and J. C. Asensi, Ind. Eng. Chem. Res., 2007, 46, 4572-4576.

3 M. Errico and B. G. Rong, Sep. Purif. Technol., 2012, 96, 58-67. 4 H. Habaki, O. Tabata, J. Kawasaki and R. Egashira, J. Chem. Eng. Jpn., 2010, 43(2), 214-223.

5 P. Christen, M. Minier and H. Renon, Biotechnol. Bioeng., 1990, 36, 116-123.

6 Z. Lei, H. Wang, R. Zhou and Z. Duan, Chem. Eng. J., 2002, 87, 149-156.

7 A. Pereiro, J. Araújo, J. Esperancca, I. Marrucho and L. Rebelo, J. Chem. Thermodyn., 2012, 46, 2-28.

$8 \mathrm{~W}$. Arlt, M. Seiler, C. Jork and T. Scheiner, Ionic Liquids as Selective Additives for the Separation of Close-Boiling or Azeotropic Mixtures, PCT Int. Appl. WO 0274718 A2, 2002.

9 C. Jork, M. Seiler, Y.-A. Beste and W. Arlt, J. Chem. Eng. Data, 2004, 52, 852-857.

10 R. P. Swatloski, J. D. Holbrey and R. D. Rogers, Green Chem., 2003, 5, 361-363.

11 M. Freemantle, An Introduction to Ionic Liquids, Royal Society of Chemistry, 2009.

12 A. P. Abbott, D. Boothby, G. Capper, D. L. Davies and R. K. Rasheed, J. Am. Chem. Soc., 2004, 126, 9142-9147.

13 M. Francisco, A. van den Bruinhorst and M. C. Kroon, Green Chem., 2012, 14(8), 2153-2157.

14 Q. Zhang, K. D. O. Vigier, S. Royer and F. Jérôme, Chem. Soc. Rev., 2012, 41, 7108-7146.

15 M. Francisco, A. van der Bruinhorst and M. Kroon, Angew. Chem., Int. Ed., 2013, 52, 3074-3085.

16 M. Francisco, A. van der Bruinhorst, L. F. Zubier, C. J. Peters and M. Kroon, Fluid Phase Equil., 2013, 340, 77-84.

17 O. Redlich and A. Kister, Ind. Eng. Chem., 1948, 40, 345-348.

18 H. Rodríguez and J. F. Brennecke, J. Chem. Eng. Data, 2006, 51, 2145-2155. 
19 U. Domanska and M. Królikowska, J. Solution Chem., 2012, 41, 1422-1445.

20 C. Antoine, C. R. Acad. Sci., 1888, 107, 681.

21 H. Renon and J. M. Prausnitz, AIChE J., 1968, 14, 135-144.

22 P. T. Ngema, Separation Processes for High Purity Ethanol Production, 2010.

23 A. V. Orchilles, P. J. Miguel, E. Vercher and A. MartínezAndreu, J. Chem. Eng. Data, 2010, 55, 1669-1674.
24 W. Geng, L. Z. Zhang, D. S. Deng, Y. Ge and J. B. Li, J. Chem. Eng. Data, 2010, 55, 1679-1683.

25 L. Z. Zhang, Y. Ge, D. X. Ji and J. B. Li, J. Chem. Eng. Data, 2009, 54, 2322-2329.

26 Y. Ge, L. Z. Zhang, X. C. Yuan, W. Geng and J. B. Li, J. Chem. Thermodyn., 2008, 40, 1248-1252.

27 D. Sunita, G. Shamla and P. L. V. N. Saichandra, National Conference, CHEMCON, 2010. 